\title{
Intrinsic Subdivision with Smooth Limits for Graphics and Animation
}

\author{
Johannes Wallner and Helmut Pottmann
}

This paper demonstrates the definition of subdivision processes in nonlinear geometries such that smoothness of limits can be proved. We deal with curve subdivision in the presence of obstacles, in surfaces, in Riemannian manifolds, and in the Euclidean motion group. We show how to model kinematic surfaces and motions in the presence of obstacles via subdivision. As to numerics, we consider the sensitivity of the limit's smoothness to sloppy computing.

Categories and Subject Descriptors: I.3.5 [Computer Graphics]: Computational Geometry and Object Modeling

General Terms: Algorithms

Additional Key Words and Phrases: nonlinear subdivision, geodesic subdivision, subdivision in surfaces, motion design, obstacles, smoothness, computing in nonlinear geometries.

\section{INTRODUCTION}

\subsection{Background and previous work}

Subdivision is an appealing way of defining a continuous object by a discrete approximation, and a curve or surface generated by subdivision has many desirable properties. It is therefore not surprising that subdivision has been extended to nonlinear spaces.

Spheres have been considered by [Kim et al. 1995; Ramamoorthi and Barr 1997; Jüttler and Wagner 2002], always having in mind that the points of the quaternion unit sphere represent the matrix parts of rigid body motions. Also with a view toward motion design, Sprott and Ravani [1997] extended B-spline algorithms to Lie groups. Nonlinear interpolatory schemes in Lie groups were constructed from linear schemes by [Donoho 2001], and used in various applications such as in smoothly interpolating a motion given at discrete instances. This material is also contained in [Ur Rahman et al. 2005], which gives a comprehensive treatment of multiscale representation for Lie-valued data and the corresponding nonlinear subdivision rules employed. We would also like to mention the nonlinear median-interpolating subdivision schemes and their extensions [Xie and Yu 2005; Oswald 2004], interpolatory schemes based on the idea of essential non-oscillation [Cohen et al. 2003], and nonlinear geometry driven schemes [Marinov et al. 2004], whose construction and analysis is different from the schemes used in this paper.

When subdividing in inherently nonlinear environments, the following problems arise:

- The definition of many linear subdivision rules employs affine combination of points. It is necessary to find a replacement for this basic way of creating new points.

- Results guaranteeing certain desirable properties (smoothness, for example) of the limits generated by nonlinear rules are harder to come by. Numerical evidence however says that they must be there.

- Computing in curved geometries often requires discretization, and sometimes also iterative processes too time-consuming to be pursued 'until they converge', as it is sometimes put. Quantitative information on the consequences of inaccuracies introduced by incomplete computation is usually not available.

It is the aim of this paper to demonstrate how certain ways of mimicking linear subdivision rules in

Authors' address: Institute of Discrete Mathematics and Geometry, Vienna University of Technology.

Email \{wallner,pottmann\}@geometrie.tuwien.ac. at 
nonlinear geometries leads to nonlinear subdivision schemes with provably smooth limits.

For linear subdivision, the smoothness analysis in many cases can be considered complete. We do not attempt to review the existing literature here, but refer to the surveys [Dyn 1992; Warren and Weimer 2001; Kobbelt 2002; Sabin 2002]. Smoothness results for nonlinear subdivision schemes have been shown by L. Noakes $[1997 ; 1998 ; 1999]$ : the geodesic analogues of the $2^{\text {nd }}$ and $3^{\text {rd }}$ degree Lane-Riesenfeld algorithm in Riemannian manifolds possess smooth limit curves with Lipschitz derivative. Recently some general smoothness results have been given for a wide class of nonlinear schemes which arise as perturbations of linear schemes [Wallner and Dyn 2005; Wallner 2006a]. It is that work which serves as a theoretical basis of this paper. Some examples are briefly mentioned already in [Wallner and Dyn 2005] in order to illustrate the theory. They are mentioned again in the present paper, whose contributions to nonlinear subdivision are a long list of examples where nonlinear subdivision schemes are useful in Computer Graphics; obstacle subdivision; intrinsic shape properties of geodesic subdivision; the use of configuration spaces for subdividing poses and the treatment of obstacles. A theoretical result contained in the present paper concerns verification of smoothness for sloppy computations.

\subsection{Overview}

This paper is organized as follows: Section 2 gives a long list of examples of nonlinear subdivision schemes which are constructed in analogy to linear schemes by the means available in various nonlinear geometries. The nonlinear schemes constructed in this way can be seen as perturbations of linear ones. In a few cases we also discuss numerical issues.

Section 3 verifies that the available theory applies to the subdivision rules defined in the previous section. We invoke general results concerning the proximity of subdivision rules and verify that the proximity conditions are fulfilled, so that the limits are $C^{1}$ and in some cases even $C^{2}$. A proof of convergence from proximity is also included.

Section 4 deals with the problem of incomplete computation of geodesics in surfaces. It turns out that a wide class of iterative algorithms used for evaluating certain nonlinear subdivision rules is quite resistant to sloppy usage.

As we emphasize the smoothness of limits, we mostly speak about smooth geometries like parametrized surfaces or level sets. After discretization (e.g. when using triangulated surfaces) the question of smoothness does no longer make sense in the strict mathematical sense. Even so, it is important to know that when computing approximately by means of a triangulation, the ideal geometric object one tries to approximate is smooth. On the other hand, there are geometries which are not discretized at all, e.g. the Euclidean motion group, and for which smoothness has the precise mathematical meaning.

\subsection{Notation}

We use the symbol $\mathbf{p}$ for a polygon, which may be either closed or open, and is a synonym for 'list of vertices'. We write $\mathbf{p}_{i}$ for the $i$-th vertex. A binary subdivision scheme $S$ maps a polygon $\mathbf{p}$ to the polygon $S \mathbf{p}$, consisting of the points $(S \mathbf{p})_{i}$, or $S \mathbf{p}_{i}$ for short. The result of two rounds of subdivision applied to $\mathbf{p}$ is denoted by $S^{2} \mathbf{p}$, and so on. The symbol $S^{\infty} \mathbf{p}$ means the limit curve. We express linear subdivision rules in terms of the affine average operator

$$
\operatorname{av}_{t}(\mathbf{a}, \mathbf{b})=\mathbf{a}+t(\mathbf{b}-\mathbf{a}) .
$$

For instance, the $n$-th degree B-spline subdivision rule $B_{n}(n \geq 2)$ is recursively defined by

$$
B_{2} \mathbf{p}_{2 i}=\operatorname{av}_{1 / 4}\left(\mathbf{p}_{i}, \mathbf{p}_{i+1}\right), \quad B_{2} \mathbf{p}_{2 i+1}=\operatorname{av}_{3 / 4}\left(\mathbf{p}_{i}, \mathbf{p}_{i+1}\right), \quad B_{n} \mathbf{p}_{i}=\operatorname{av}_{1 / 2}\left(B_{n-1} \mathbf{p}_{i}, B_{n-1} \mathbf{p}_{i+1}\right) .
$$

$B_{2}$ is Chaikin's rule. For an illustration of $B_{2}$ and $B_{3}$, see Fig. 7. The polygons $\left(B_{n}\right)^{j} \mathbf{p}$ converge to the $n$-th degree B-spline curve for which the polygon $\mathbf{p}$ and, indeed, any polygon $\left(B_{n}\right)^{j} \mathbf{p}$, is a control polygon. 


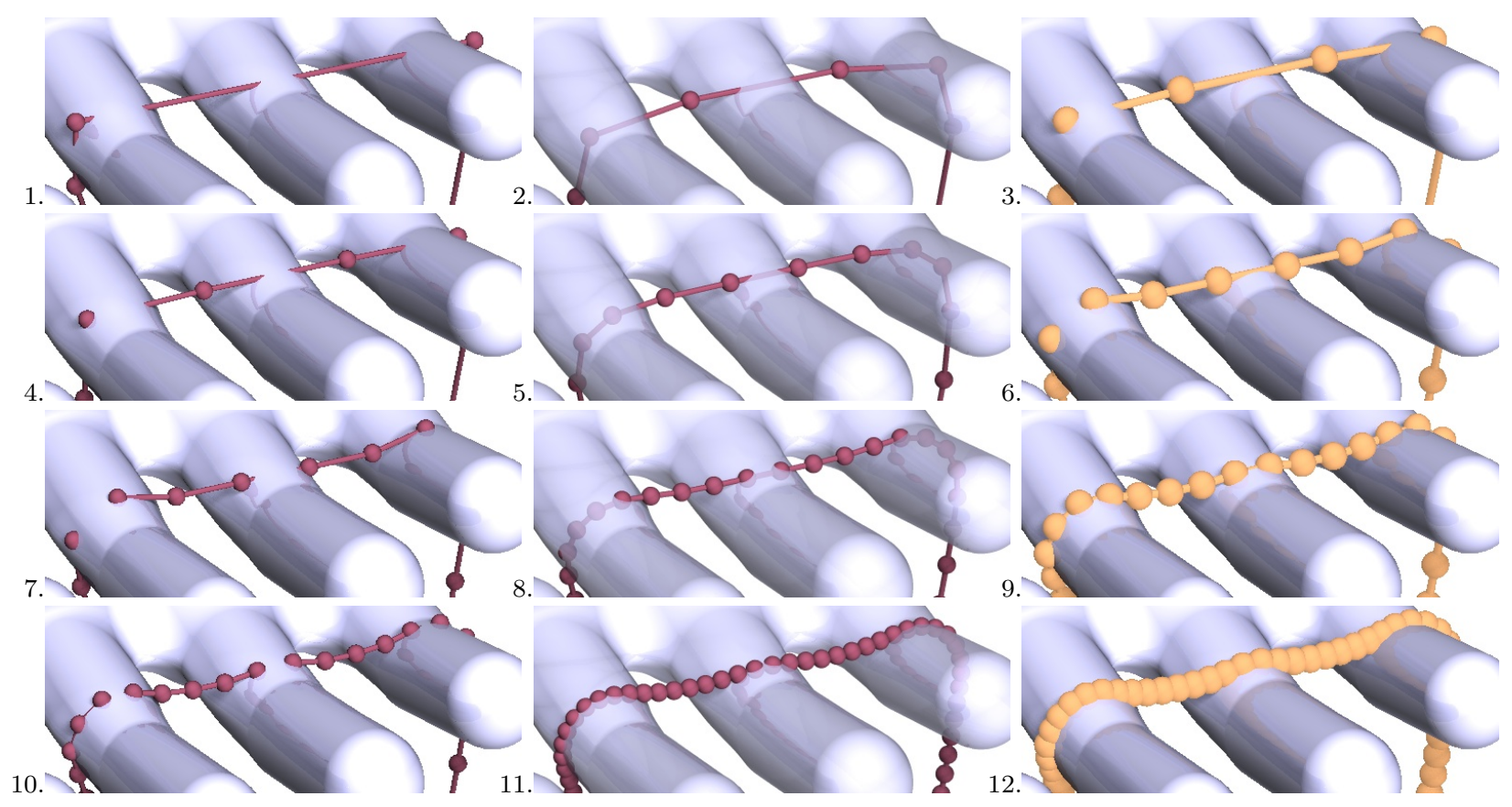

Fig. 1. Perturbing a linear subdivision rule by an obstacle and other influences. The sequence of images is described in Sec. 2.1 .

Another prominent rule is the interpolatory four-point scheme of [Dyn et al. 1987]:

$$
S \mathbf{p}_{2 i}=\mathbf{p}_{i}, \quad S \mathbf{p}_{2 i+1}=\operatorname{av}_{1 / 2}\left(\operatorname{av}_{-2 w}\left(\mathbf{p}_{i}, \mathbf{p}_{i-1}\right), \operatorname{av}_{-2 w}\left(\mathbf{p}_{i+1}, \mathbf{p}_{i+2}\right)\right) \quad \text { with } 0<w<1 / 8 .
$$

\section{EXAMPLES}

A long list of examples demonstrates how to modify linear curve subdivision rules so that they work in the presence of obstacles, within surfaces, and in the Euclidean motion group. Smoothness is discussed in Sec. 3.

\subsection{Subdivision in the presence of obstacles}

Designing curves in the presence of obstacles is an old problem, and we do not attempt to give an overview about the literature. We use a simple method which in the end amounts to perturbing linear subdivision rules such that smoothness is preserved.

We work in three-dimensional Euclidean space minus a certain forbidden domain (the obstacle, denoted by $O$ ). When applying a subdivision scheme $S$ to a polygon $\mathbf{p}$, then the polygon $S \mathbf{p}$ might have points which lie in $O$, even if $\mathbf{p}$ was outside $O$. Fig. 1.1 shows an example: The vertices $\mathbf{p}_{i}$ are outside an obstacle (blue). The result $S \mathbf{p}$ of subdivision can be seen in Fig. 1.2. Here $O$ is the set $\{\mathbf{x} \mid F(\mathbf{x})<c\}$ where $F$ is a blob function, and $S$ is the B-spline scheme $B_{4}$ defined by Equ. (2).

In order to define a subdivision rule $T$ which respects the forbidden domain $O$, we assume that there is a mapping $P$ which moves a point in the interior of $O$ to the obstacle boundary $\partial O$, and let $T \mathbf{p}_{i}=P S \mathbf{p}_{i}$ in case $S \mathbf{p}_{i}$ lies in $O$ and $T \mathbf{p}_{i}=S \mathbf{p}_{i}$ otherwise.

Later, when discussing smoothness, properties of this projection onto the boundary will become relevant. In our example, $\partial O$ is the level set $F(\mathbf{x})=c$, and $P$ is the gradient flow of $F$ towards that level set. The result looks like Fig. 1.3.

Actually the subdivision scheme $T$ used for this particular example was constructed in a slightly different 
Fig. 2. The left hand image shows the control polygon of provably smooth spaghetti. The right hand figure illustrates this smooth limit by depicting the result of 5 rounds of nonlinear Bspline subdivision avoiding the interior of the hand, with added gravity. For details, see Sec. 2.1.

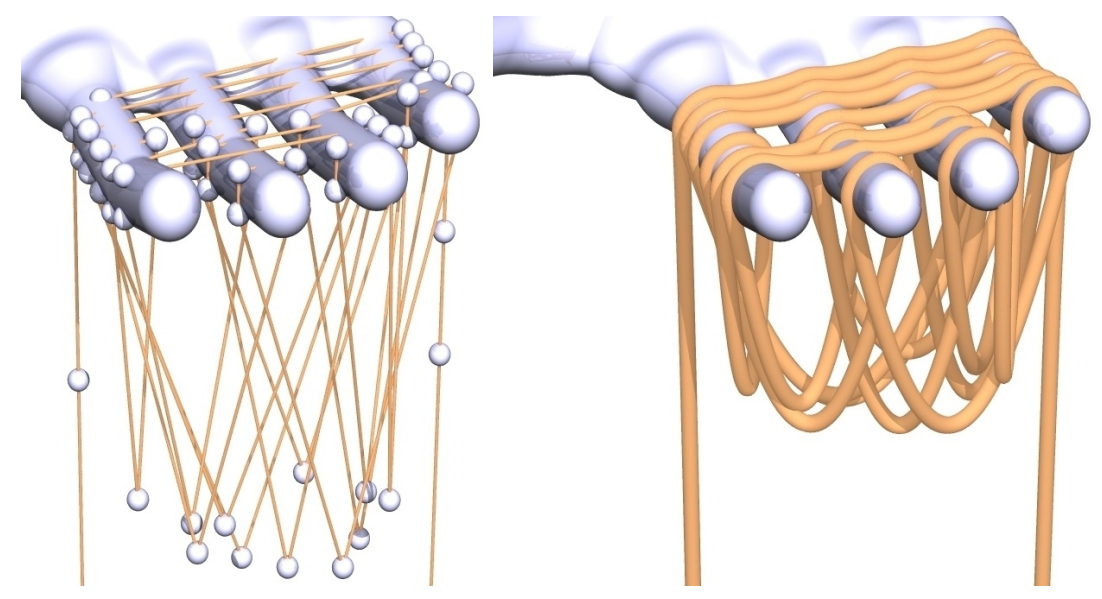

way: In the recursive definition of $B_{4}$ given by Equ. (2) every averaging is immediately followed by a projection onto the boundary of the obstacle, should the result of averaging lie in $O$. It turns out that both ways of perturbing a linear scheme lead to smooth limit curves.

We want to show that for applications one might want to modify a given subdivision scheme for a variety of reasons and in many different ways. In our case we produce an image of a handful of spaghetti, which in the real world is under the influence of gravity. Thus we add the following perturbation: Before again subdividing with $B_{4}$, we decrease the $z$-coordinate of each point by a small amount. Here we choose

$$
\mathbf{p}_{i} \rightarrow \mathbf{p}_{i}-C \cdot\left\|\mathbf{p}_{i+1}-\mathbf{p}_{i}\right\|^{2} \cdot(0,0,1) .
$$

This small movement of vertices is hardly visible. The result is shown by Fig. 1.4.

The sequence of images of Fig. 1.1-12 documents several rounds of this iterative process - an orange polygon first experiences a gravity-like perturbation, is further subdivided linearly for illustration (shown with transparent obstacle), and nonlinearly (shown again in orange). Subdivision of a longer polygon $\mathbf{p}$ is shown in Fig. 2. Note that this procedure neither simulates the actual statics of pasta, nor does it take self-intersections into account. It intends to demonstrate two possible perturbations which may be inflicted on a linear subdivision rule.

\subsection{Geodesic Subdivision}

If the vertices $\mathbf{p}_{i}$ of a polygon are contained in a surface $M$, it is in general not true that after subdivision with a linear scheme $S$, the vertices $S \mathbf{p}_{i}$ are also contained in $M$. It is therefore impossible to use such subdivision schemes directly if one wants to work entirely in $M$. One way of dealing with this problem is to employ geodesic analogues of linear rules, which are the topic of the next paragraphs.

2.2.1 Curve Subdivision within Surfaces. An idea which occurs naturally when looking for an intrinsic equivalent of linear subdivision rules is to replace straight lines by geodesic lines, which are the shortest paths between points on the surface [do Carmo 1992]. Because of the computational difficulties involved, geodesic subdivision defined in this way is useful only in special circumstances. The idea however is important and is explained now in more detail: If $\mathbf{c}(t)$ is a constant speed parametrization of a geodesic line emanating from $\mathbf{a}$ and ending in $\mathbf{b}$, then a geodesic average is defined by

$$
\mathbf{c}(0)=\mathbf{a}, \mathbf{c}(1)=\mathbf{b} \Longrightarrow \mathrm{g}-\mathrm{av}_{t}(\mathbf{a}, \mathbf{b})=\mathbf{c}(t) .
$$

Because of the symmetry relation $g-\operatorname{av}_{t}(\mathbf{a}, \mathbf{b})=\mathrm{g}-\mathrm{av}_{1-t}(\mathbf{b}, \mathbf{a})$, "g-av" is a a satisfactory intrinsic replacement of "av". We replace "av" by "g-av" in the definition the B-spline rule $B_{n}$ in Equ. (2) and thus get a geodesic 
1.

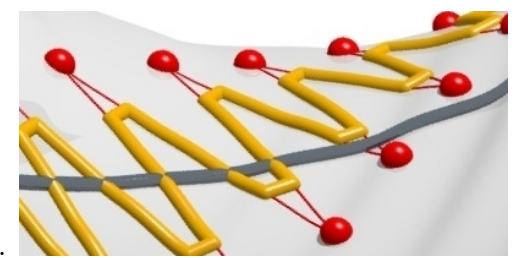

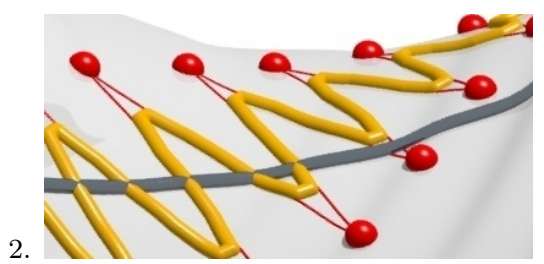

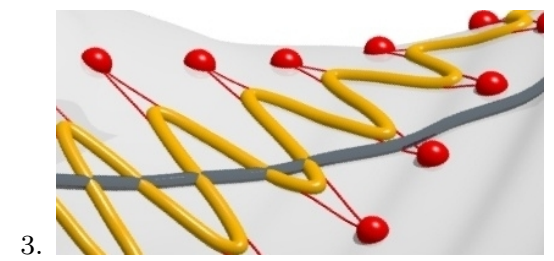

Fig. 3. Geodesic Subdivision. 1-3: Subdivision by the geodesic analogue $T_{2}$ of Chaikin's rule $B_{2}$. The polygon $\mathbf{p}$ is shown in red and, from left to right, $T_{2} \mathbf{p}, T_{2}^{2} \mathbf{p}, T_{2}^{\infty} \mathbf{p}$ in yellow. The variation diminishing property of geodesic B-splines is illustrated by means of a grey geodesic line.

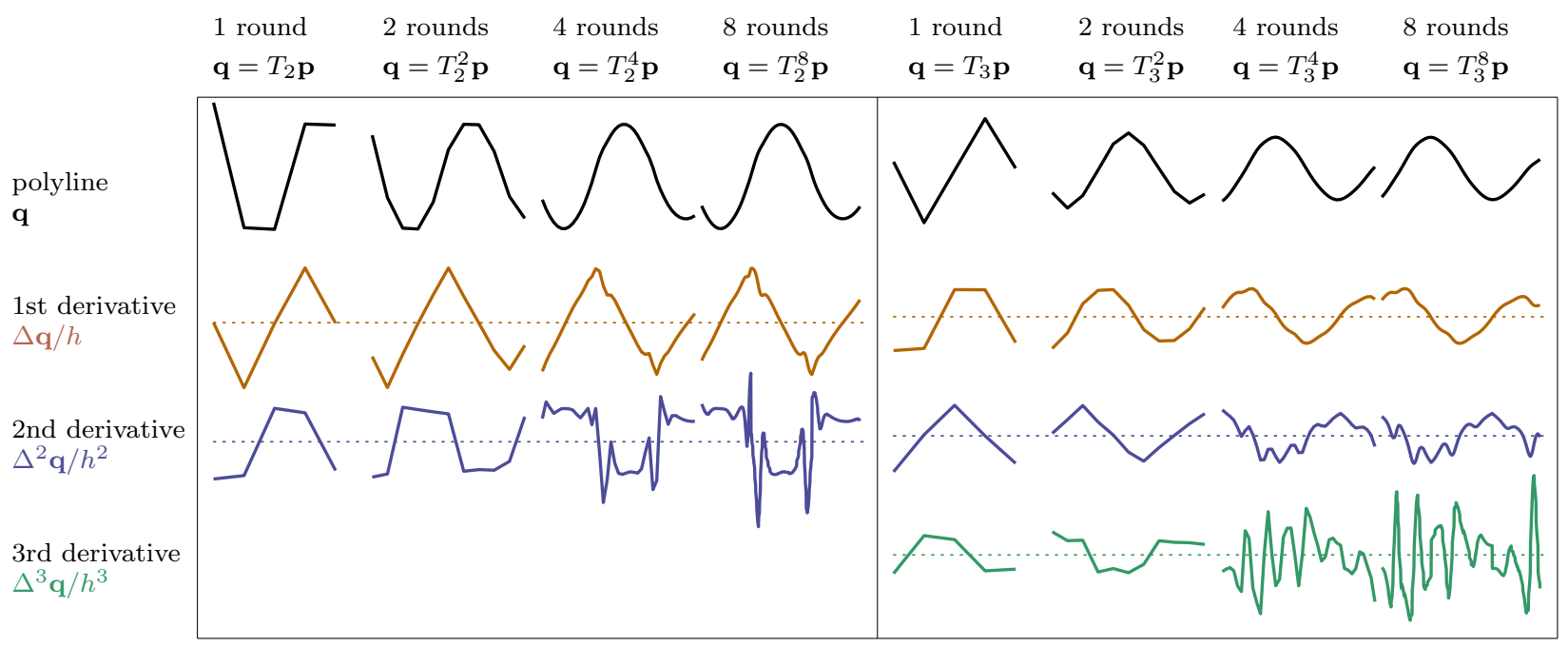

Fig. 4. Visualization of smoothness of the geodesic Chaikin rule " $T_{2}$ " in the wriggly surface of Fig. 3 (left) and of the cubic geodesic B-spline rule " $T_{3}$ " (right). The figures illustrate numerical smoothness after several rounds of subdivision. Each small figure depicts a diagram of the first coordinate of the points under consideration, the diagrams for the second and third coordinate being similar. Scaling in each figure depends only on the order of differentiation, so that derivatives of different polylines are comparable. From the blue figures it is obvious that for the Chaikin rule $T_{2}$, the second numerical derivative no longer appears to be continuous, while for the cubic rule, the third numerical derivative (green) is the first to show this behaviour.

B-spline rule of degree $n$, which is illustrated in Fig. 3

Generically, the shortest geodesic which contains two given points $\mathbf{a}, \mathbf{b}$ is unique, and for a given surface there is always a certain positive distance $\delta$ such that uniquenes holds in case $\|\mathbf{a}-\mathbf{b}\|<\delta$. Another problem which might arise is that in a non-complete surface it can happen that there is no unbroken geodesic which joins two given points $\mathbf{a}, \mathbf{b}$. These problems are unlikely to occur at all, as we usually subdivide polygons whose vertices are close together. 


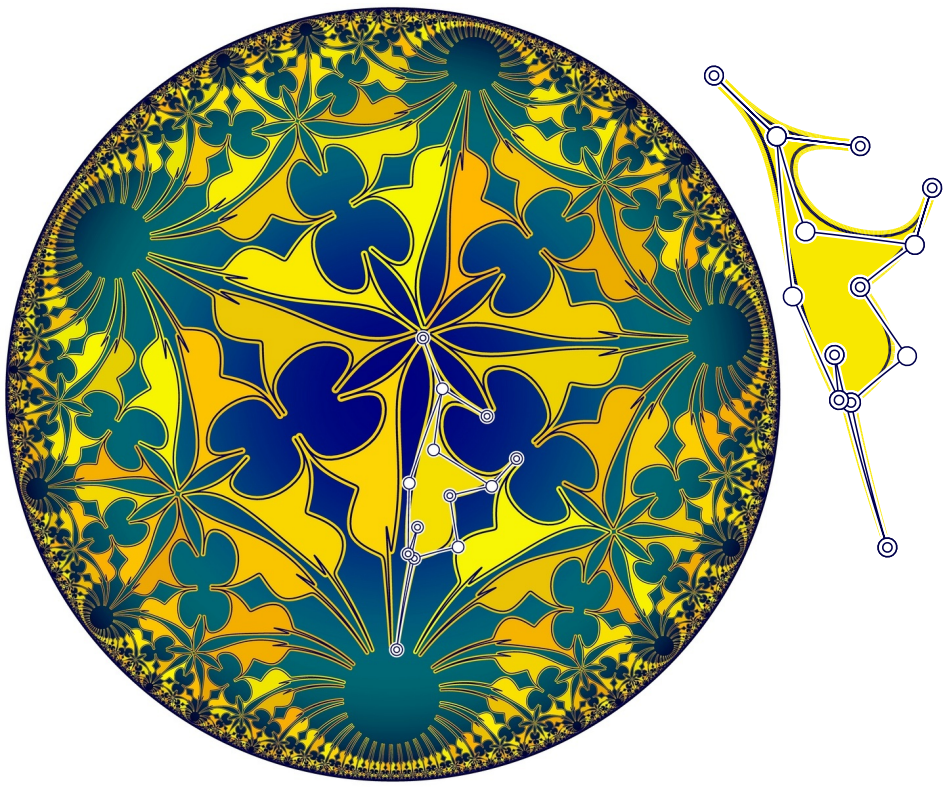

Fig. 5. Hyperbolic swordfish (Geodesic analogue of B-Spline subdivision $B_{3}$ with $C^{2}$ limit curves). One hyperbolic control polygon is also shown.
2.2.2 Subdivision in Riemannian manifolds. The word Riemannian manifold means a manifold (which for the purposes of Computer Graphics is realized as a piece of $\mathbb{R}^{d}$ or a parametrized surface or a triangulation), where the way of computing the scalar product of two tangent vectors attached to the same point is not the one of ambient space, but is allowed to change smoothly with the point quite independent of the way the manifold lies in some other space. Of course we might as well use the scalar product of ambient space, so every surface in the ordinary sense is a Riemannian manifold. It turns out that the theory dealing with smoothness of limits extends easily to Riemannian manifolds, so for the sake of completeness we give an example of subdivision in terms of geodesics of a Riemannian manifold.

The Poincaré disk model of hyperbolic geometry (here thought to be contained in the unit disk of $\mathbb{R}^{2}$, see Fig. 5) is a fascinating geometry which appears in Computer Graphics e.g. because it is suitable for visualization of surface-like data which will not fit well into the Euclidean plane. Geodesics are easily computable [Alekseevskij et al. 1993] - they appear as circles which intersect the boundary of the hyperbolic disk orthogonally. The geodesic midpoint $g-\mathrm{av}_{1 / 2}(\mathbf{a}, \mathbf{b})$ of points $\mathbf{a}, \mathbf{b}$ is computed by

$$
\begin{aligned}
& \operatorname{g}^{-a_{1 / 2}}(\mathbf{a}, \mathbf{b})=\psi\left(\frac{\phi(\mathbf{a})+\phi(\mathbf{b})}{\|\phi(\mathbf{a})+\phi(\mathbf{b})\|}\right), \quad \text { where } \quad\|\mathbf{x}\|^{2}=x_{1}^{2}-x_{2}^{2}-x_{3}^{2}, \\
& \phi(\mathbf{x})=\frac{1}{1-\mathbf{x}^{2}}\left(1+\mathbf{x}^{2}, 2 x_{1}, 2 x_{2}\right), \quad \psi(\mathbf{x})=\frac{1}{1+x_{1}}\left(x_{2}, x_{3}\right) .
\end{aligned}
$$

Equ. (6) is sufficient for defining hyperbolic B-spline subdivision analogous to Equ. (2). because

$$
\mathrm{g}-\mathrm{av}_{1 / 4}(\mathbf{a}, \mathbf{b})=\mathrm{g}-\mathrm{av}_{1 / 2}\left(\mathbf{a}, \mathrm{g}-\mathrm{av}_{1 / 2}(\mathbf{a}, \mathbf{b})\right), \quad \mathrm{g}-\mathrm{av}_{3 / 4}(\mathbf{a}, \mathbf{b})=\mathrm{g}-\mathrm{av}_{1 / 4}(\mathbf{b}, \mathbf{a}) .
$$

The curves which appear in Fig. 5 are the result of hyperbolic B-spline subdivision analogous to $B_{3}$.

2.2.3 Intrinsic shape properties of geodesic schemes. If a planar subdivision rule $S$ works by corner cutting (the B-spline schemes do), then it enjoys the variation diminishing property: A straight line intersects the edges of each polygon $S^{i} \mathbf{p}$ at least as often as it intersects the edges of the polygon $S^{j} \mathbf{p}$, if $j>i(j=\infty$ and $i=0$ are allowed). This follows directly from the fact that two line segments have at most one intersection point.

Therefore geodesic subdivision rules in a 2-dimensional surface or manifold have the same property, provided geodesic segments intersect in one point only. This is not the case in general, but can be guaranteed for any compact surface if the shorter of the two segments in question is not longer than a certain constant, which depends on the intrinsic curvature of the surface [do Carmo 1992]. This geodesic variation diminishing property is illustrated by Fig. 3. 
2.2.4 Numerics of geodesic lines. Here we discuss some issues related to discretization and computation of geodesics in surfaces. Fast computation of geodesics is a topic of its own (see e.g. [Mémoli and Sapiro 2001; 2005; Surazhsky et al. 2005]). We show how geodesics can be computed in principle, and we collect facts which in Section 4 help to analyze the results of incomplete computation.

In the following we discuss a well known infinitesimal characterization of geodesic lines "(i)" and a characterization of arc length minimizing geodesics in terms of energy "(ii)": (i) If a geodesic c $(t)$ in a surface $M$ is traversed with constant speed, the vector $\mathbf{c}^{\prime \prime}(t)$ is orthogonal to $M$; (ii) minimal geodesics traversed with constant speed are precisely the minimizers of the energy functional $\int\left\|\mathbf{c}^{\prime}(t)\right\|^{2} d t$. When representing the curve $\mathbf{c}(t)$ by a polygon $\mathbf{p}_{0}, \mathbf{p}_{1}, \ldots, \mathbf{p}_{k}$, a classical discretization of this is as follows: (i): the second forward difference $\Delta^{2} \mathbf{p}_{i-1}=\mathbf{p}_{i+1}-2 \mathbf{p}_{i}+\mathbf{p}_{i-1}$ is orthogonal to $M$ in the point $\mathbf{p}_{i}$; (ii): the discrete energy $\sum\left\|\mathbf{p}_{i+1}-\mathbf{p}_{i}\right\|^{2}$ is minimal. It is well known that polygons with property (ii) also satisfy (i).

Discretization (i) is suitable for solving the initial value problem, i.e., computing a discrete geodesic with given endpoint $\mathbf{a}$ and tangent vector $\mathbf{v}$ : We choose $\mathbf{p}_{0}=\mathbf{a}$, determine $\mathbf{p}_{1}$ as close as possible to $\mathbf{a}+\mathbf{v}$, and recursively find $\mathbf{p}_{i+1}$ such that the condition on $\Delta^{2} \mathbf{p}_{i-1}$ is fulfilled, which means that $\mathbf{p}_{i+1}$ is the intersection point of the given surface with a line orthogonal to the surface at $\mathbf{p}_{i}$ and and passing through the point $\mathbf{p}_{i}+\left(\mathbf{p}_{i}-\mathbf{p}_{i-1}\right)$. This is a numerical "Euler polygon" method for solving an ODE, so the discretization error, i.e., the difference between the point $\mathbf{p}_{i}$ and an actual geodesic, is bounded by a constant times the step size squared. The precise inequality is given in Section 4.2, Equation (17).

Discretization of type (ii) is suitable for solving the boundary value problem, i.e., computing a discrete geodesic with given endpoints $\mathbf{a}$ and $\mathbf{b}$. We let $\mathbf{p}_{0}=\mathbf{a}, \mathbf{p}_{k}=\mathbf{b}$, and choose the remaining points somehow in between. It is elementary that moving the points $\mathbf{p}_{i}(0<i<k)$ in direction $\Delta^{2} \mathbf{p}_{i-1}$ decreases energy, so it is easy to set up a gradient descent or conjugate gradient method for minimization. If $k$ is a power of two, the multigrid idea accelerates this procedure. The discretization error is the same as it was for the initial value problem. This is because property (ii) implies (i), so a polygon found with (ii) occurs among the polygons found using criterion (i).

\subsection{Projection Subdivision}

Often projections onto surfaces are computable. Here projection does not necessarily mean orthogonal projection, but any smooth mapping which maps onto the surface and which leaves the points of the surface fixed. Especially if the surface in question is a level set $F(\mathbf{x})=c$, a projection is provided by any procedure which moves a point towards the level set and whose output depends smoothly on the input.

If a projection $P$ is given, we modify a given subdivision scheme $S$ as follows: An analogous projection scheme " $T$ " is defined by $T \mathbf{p}_{i}=P S \mathbf{p}_{i}$. In fact in our detailed discussion of subdivision in the presence of obstacles we already encountered such a projection. The difference to the obstacle setting is that here the projection is applied to all points, not only those inside a forbidden domain.

If a subdivision scheme happens to be defined by a certain number of averaging steps, we might apply the projection onto the surface every time after averaging. This leads to a different kind of analogous projection scheme. The idea of projection subdivision is especially important in the next section, which deals with motion design.

\subsection{Subdivision in the Euclidean motion group}

Like previous sections, Sec. 2.4 describes ways of defining intrinsic subdivision rules in a nonlinear environment. Here we work with Euclidean positions of a rigid body.

The position of a rigid body in space is described by a $3 \times 3$ orthogonal matrix $A$ with positive determinant and a vector of $\mathbf{a} \in \mathbb{R}^{3}$. If $\mathbf{x}$ is the coordinate vector of a point with respect to a coordinate system attached to the rigid body, then its position in space is $A \mathbf{x}+\mathbf{a}$. The set of all such matrix-vector pairs is called the Euclidean motion group $\mathrm{SE}_{3}$. Subdividing a sequence "p" of positions of a rigid body should lead to 
1.

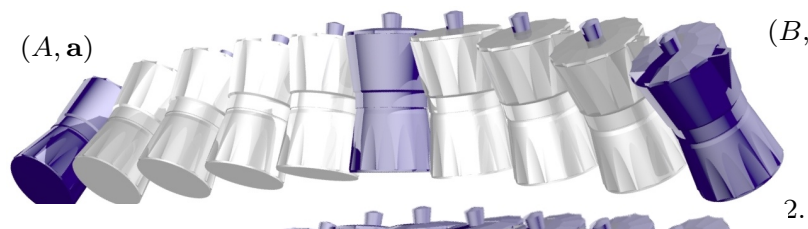

3.

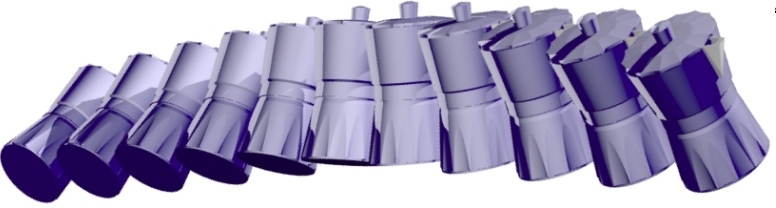

$(B, \mathbf{b})$

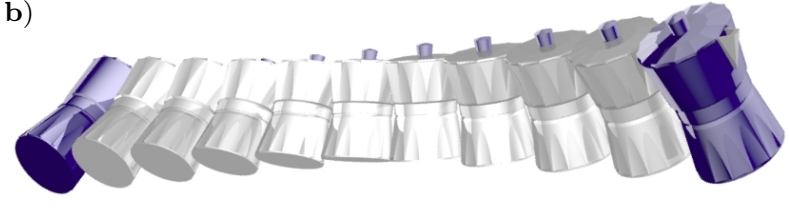

Fig. 6. 1. Group-midpoint $\left(C\left(\frac{1}{2}\right), \mathbf{c}\left(\frac{1}{2}\right)\right)$ of positions $(A, \mathbf{a}),(B, \mathbf{b})$. 2. Positions $(A, \mathbf{a})$ and $(B, \mathbf{b})$ together with averages $\operatorname{av}_{t}((A, \mathbf{a}),(B, \mathbf{b}))$. 3. Projection of averages onto $\mathrm{SE}_{3}$.

ever denser sequences $S \mathbf{p}, S^{2} \mathbf{p}$ of positions and, in the limit, to a continuous motion $S^{\infty} \mathbf{p}$. There are two straightforward ways of defining subdivision in the set of positions - one similar to geodesic subdivision in a surface, the other one being a kind of projection subdivision. They are described also in [Wallner and Dyn 2005] - here we briefly repeat the constructions in Sections 2.4.2 and 2.4.1. We subsequently extend the method to motions in the presence of obstacles, kinematic surfaces, and multibody kinematic chains.

2.4.1 Helical subdivision. We consider a uniform helical motion $(C(t), \mathbf{c}(t))$ which moves position $(A, \mathbf{a})=$ $(C(0), \mathbf{c}(0))$ of a rigid body into position $(B, \mathbf{b})=(C(1), \mathbf{c}(1))$. It is illustrated in Fig. 6.1 and can be interpreted as a curve in the motion group $\mathrm{SE}_{3}$. The helical motion which joins given positions $(A, \mathbf{a})$ and $(B, \mathbf{b})$ is not unique, but it is tacitly understood that we always choose one with the smallest possible angle of rotation. Then the group average of $(A, a)$ and $(B, b)$ is given by

$$
\mathrm{g}^{-\mathrm{av}_{t}}((A, \mathbf{a}),(B, \mathbf{b}))=(C(t), \mathbf{c}(t)) .
$$

Any subdivision rule expressible by the av operator has an analogous group rule, where "av" is replaced by "g-av". We now illustrate this concept by means of the B-spline rule $B_{3}$ defined by Equ. (2):

We start with a planar polygon $\mathbf{p}$ whose edges are shown in red and whose vertices are shown in blue (Fig. 7.1). We insert the vertices of $B_{2} \mathbf{p}$ (which lie on the edges of $\mathbf{p}$, Fig. 7.2) and draw an image of edges and vertices of $B_{2} \mathbf{p}$ (Fig. 7.3). On the edges of $B_{2} \mathbf{p}$, we draw the vertices of $B_{3} \mathbf{p}$ (Fig. 7.4). Fig. 7.5 shows $\mathbf{p}$ together with $B_{3} \mathbf{p}$, and Fig. 7.6 has $\mathbf{p}$ and $B_{3}^{2} \mathbf{p}$. The group analogues of these constructions are indicated with a polygon "q" of positions and the group subdivision rules $T_{2}$ and $T_{3}$ which are analogous to $B_{2}$ and $B_{3}$ (Fig. 8.1-6). Instead of the edges of a polygon we show the helical motions which connect the vertices. The blue positions are scaled by a factor of 1.2 for better visibility.

2.4.2 Projection subdivision in the motion group. The helical way is not the only one how subdivision in the motion group can be defined. The matrix/vector pairs $(A, \mathbf{a})$ with $A \in \mathbb{R}^{3 \times 3}$ and $\mathbf{a} \in \mathbb{R}^{3}$ without any restriction imposed on $A$ comprise the space of affine positions, which is a vector space of dimension $3 \times 3+3=12$ and which contains the Euclidean motion group as a surface of dimension six. Applying the affine average operator to Euclidean positions usually yields affine positions which are not Euclidean (Fig. 6.2), but by projecting them onto the group we get Euclidean positions again (cf. Fig. 6.3). Thus we may define projection subdivision in $\mathrm{SE}_{3}$.

It remains to define a projection onto the Euclidean motion group. This is a so-called matrix nearness problem [Higham 1989]. We follow Belta and Kumar [2002], who define a distance between affine positions: Assume that points $\mathbf{x}_{1}, \ldots, \mathbf{x}_{r}$ in which are not co-planar represent a rigid body and points $\mathbf{y}_{i}=A \mathbf{x}_{i}+\mathbf{a}$ and $\mathbf{z}_{i}=B \mathbf{x}_{i}=\mathbf{b}$ represent two different positions $\alpha=(A, \mathbf{a})$ and $\beta=(B, \mathbf{b})$, resp., of that body. We assume that $\sum \mathbf{x}_{i}=\mathbf{o}$, which means that the barycenter of the points $\mathbf{x}_{i}$ is the origin of the coordinate system attached to the rigid body in question. Then $\operatorname{dist}(\alpha, \beta)^{2}:=\sum_{i=1}^{r}\left\|\mathbf{y}_{i}-\mathbf{z}_{i}\right\|^{2}$. For given $(A$, a), we ask for the Euclidean position $(B, \mathbf{b})$ nearest to $(A, \mathbf{a})$. 

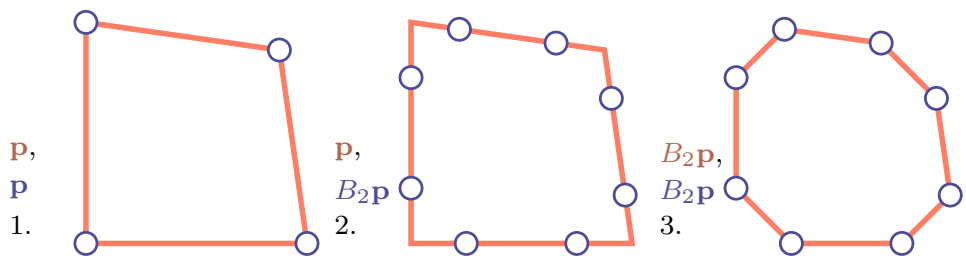

Fig. 7. Linear B-spline subdivision rules $B_{2}$ and $B_{3}$. The various combinations of a polygon $p$ and the subdivided polygons $B_{2} p$ and $B_{3} p$ correspond to the images in Fig. 8.1-8, which show analogous B-spline subdivision in the Euclidean motion group.
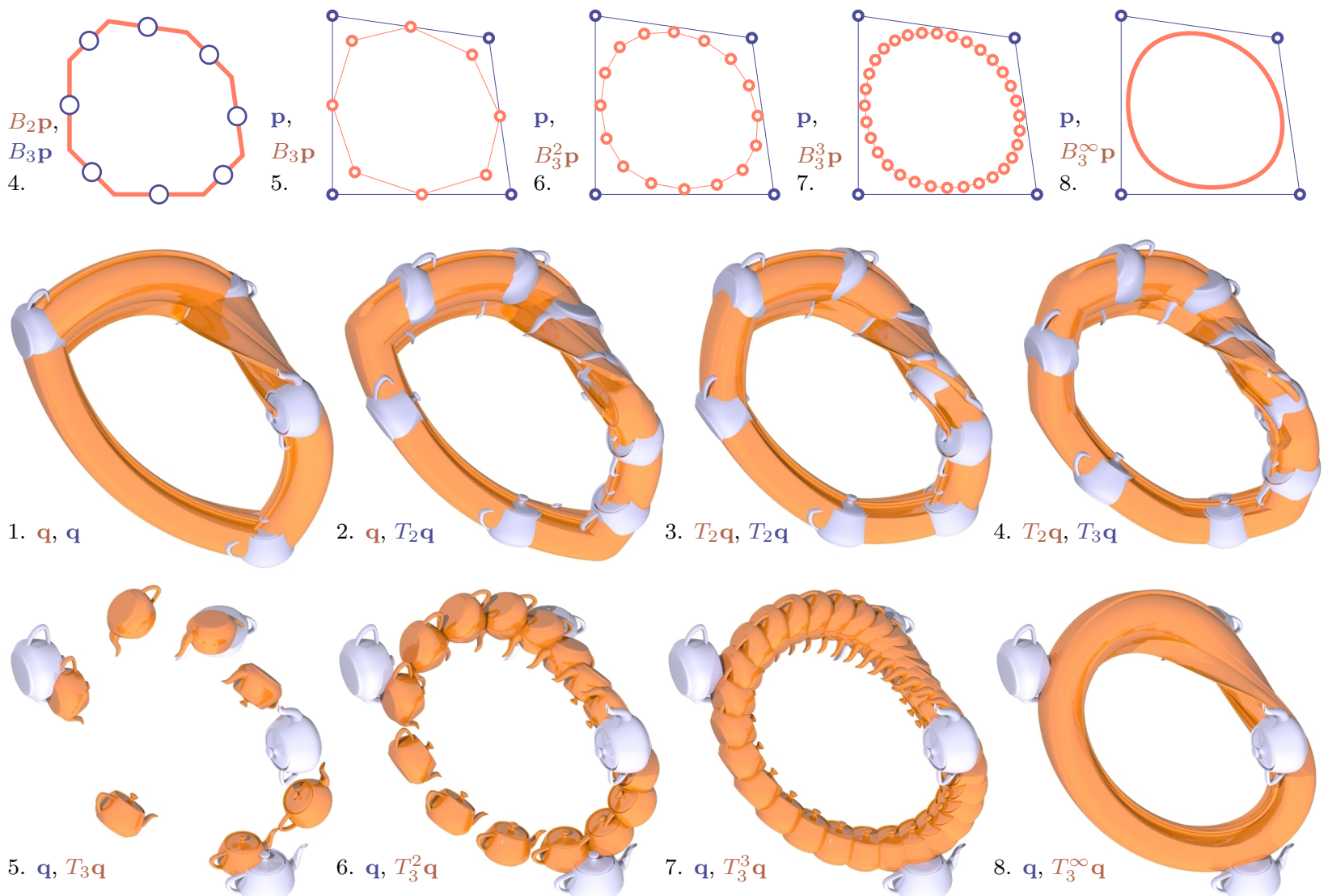

3. $T_{2} \mathrm{q}, T_{2} \mathbf{q}$

4. $T_{2} \mathbf{q}, T_{3} \mathbf{q}$
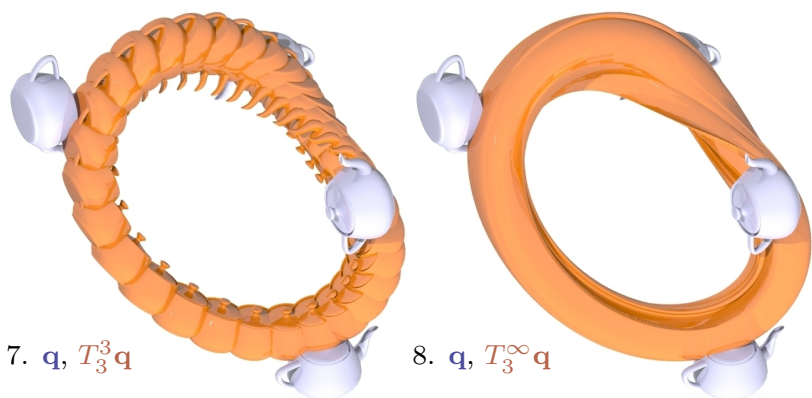

Fig. 8. 1-8: Helical subdivision $T_{2}, T_{3}$ in the Euclidean motion group analogous to Chaikin's Rule $B_{2}$ and the cubic LaneRiesenfeld rule $B_{3}$. The images correspond to Fig. 7.1-8.

The answer to this question in the case $\operatorname{det} A>0$ requires computation of the matrix $J=\sum_{i=1}^{r} \mathbf{x}_{i} \cdot \mathbf{x}_{i}^{T}$ and is given by $\mathbf{b}:=\mathbf{a}$ and $B:=P Q$, if $A J=P D Q$ is the singular value decomposition of $A J$. The definition $\mathbf{b}:=\mathbf{a}$ means that "a", the position of the barycenter of the given rigid body, is not affected by the projection. In the case $\operatorname{det} A<0$, we have $B:=P D^{\prime} Q$, where $D^{\prime}=\operatorname{diag}(-1,1,1)$, under the assumption that the singular values are sorted by size, smallest first. The distance defined above is compatible with a scalar product in $\mathbb{R}^{3 \times 3+3}$ and the projection used here is the corresponding orthogonal projection onto $\mathrm{SE}_{3}$. Thus this construction is a special case of projection subdivision (see Sec. 2.3), where the surface we project on is given by $\mathrm{SE}_{3}$.

Kinematic surfaces, such as surfaces of revolution, are traced out by the motion of a curve. Previous work on subdivision in relation to kinematic surfaces includes pp. 216-220 of [Warren and Weimer 2001], which 


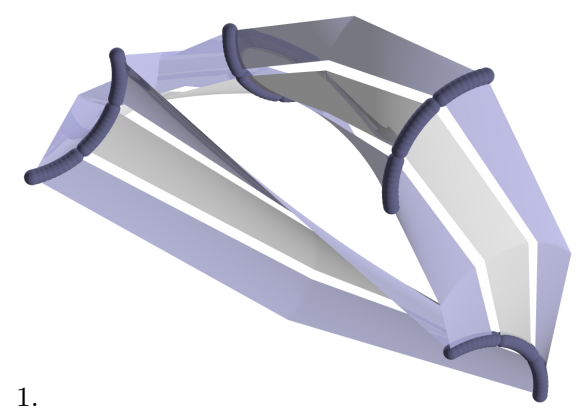

1.

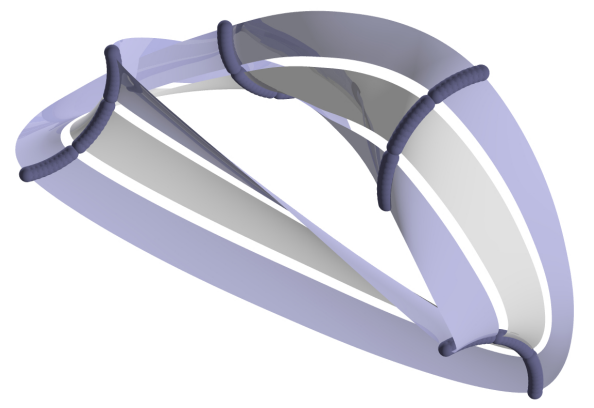

2.

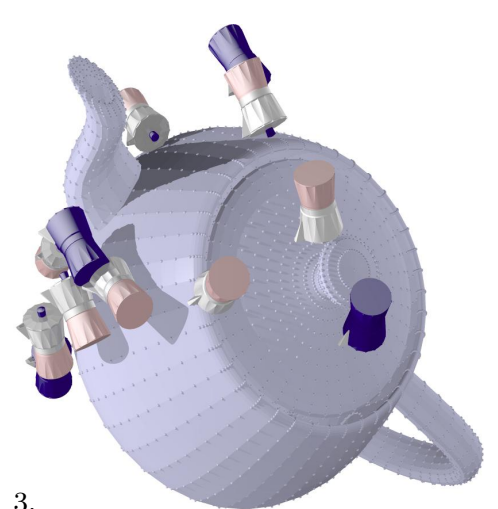

3.

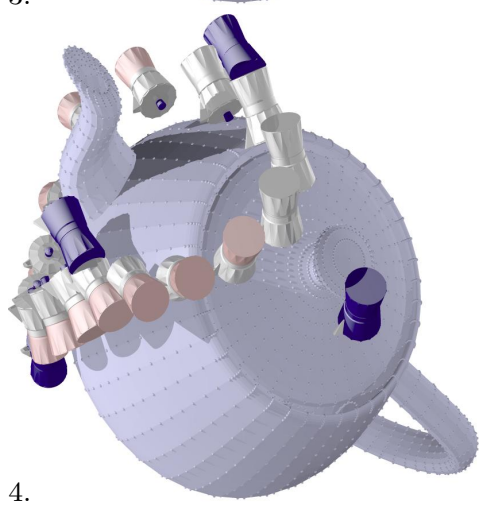

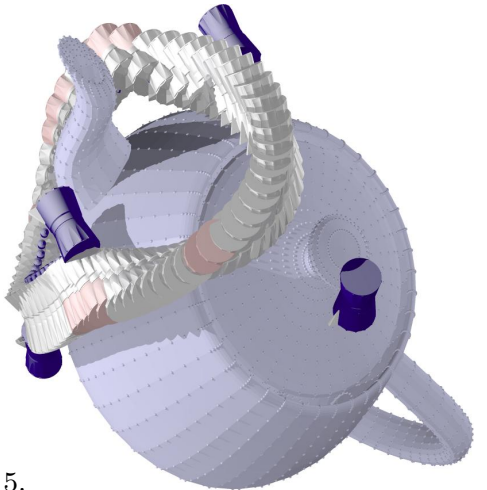

5.

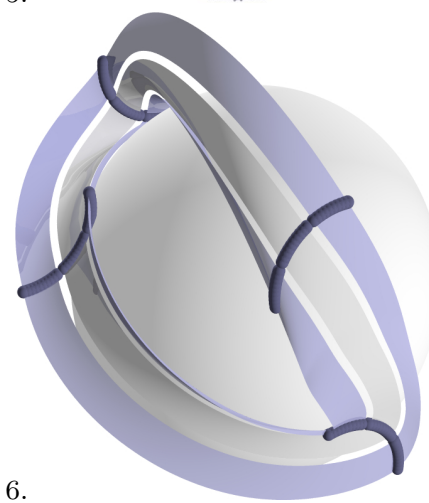

Fig. 9. Projection subdivision in the Euclidean motion group. 1-2: Kinematic surface produced by a projection scheme based on the interpolatory rule (3) and a projection onto the motion group. (1 round of subdivision and limit surface). 3-5: Avoiding the interior of a solid without gliding constraint: Control Positions of a coffee pot are shown in blue. We see 1, 2, and 4 rounds of subdivision derived from the Chaikin rule. Positions which had to be modified in the last round because they intersect with the teapot are in a different colour. 6: Kinematic surface with gliding constraint (subdivision scheme derived from the DGL rule (3)).

describe subdivision schemes converging to surfaces of revolution. We interactively design kinematic surfaces by choosing control positions of the generator curve and apply subdivision to these positions, which in the limit yields a smooth kinematic surface generated by the original curve, provided the motion generated by subdivision is smooth (Fig. 9.1-2). After the design process we might want to convert the surface into a spline representation - this benefits from the evenly spaced discrete parametrization furnished by the subdivision process.

2.4.3 Comparison and limitations of the two methods of subdivision. If subdividing a dense sequence of positions it hardly matters whether we use helical subdivision (Section 2.4.1) or projection subdivision (Section 2.4.2). A difference is noticeable only if the positions to be subdivided are wide apart.

It is always possible to construct helical motions which connect two given positions, but the shortest such motion is possibly not unique. This happens exactly if the rotation angle from the first position to the second equals $\pi$. This situation is comparable to finding the shortest arc of a great circle which connects two antipodal points on a sphere.

As to projection subdivision, the only place where the computation of averages in the nonlinear way can fail is where the computation a singular value decomposition is not unique the average $((1-t) A+t B) J$ of 


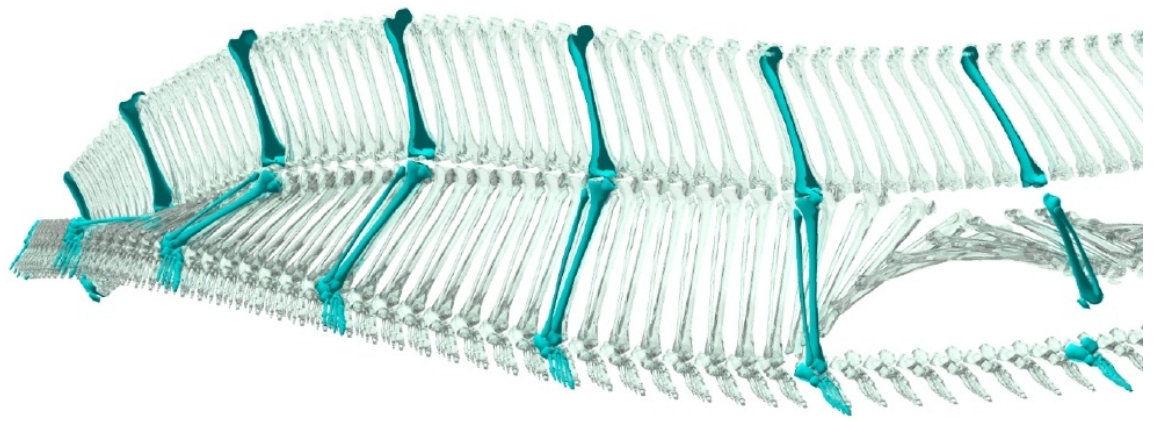

Fig. 10. Kinematic chain consisting of three bodies and two spherical joints, which suddenly cease to exist.

matrices $A, B$ is singular. It is easy to convince oneself that this happens only if the rotation described by the matrix $A^{-1} B$ is about an angle of 180 degrees, and $t=1 / 2$. So apparently both methods have about the same limitations.

2.4.4 Motions generated by subdivision which avoid obstacles. There are several ways of dealing with motions which are to avoid a forbidden domain $O$. Suppose that we start with a sequence $\mathbf{q}$ of positions. Suppose further that for each position $(A, \mathbf{a})$ of the rigid body under consideration we can find another one, denoted by $P_{O}(A, \mathbf{a})$, which is as close as possible to the original, such that the body does not enter $O$, but touches the obstacle boundary $\partial O$. A subdivision scheme $T$ acting on positions may be modified in the following way: If the body at position $T \mathbf{q}_{i}$ intersects $O$, let $\widetilde{T} \mathbf{q}_{i}:=P_{O}\left(T \mathbf{q}_{i}\right)$. Otherwise, let $\widetilde{T} \mathbf{q}_{i}:=T \mathbf{q}_{i}$. Then the subdivision scheme $\widetilde{T}$ produces motions where the given rigid body avoids the interior of $O$ (see Fig. 9.3-5). If a body is to glide on $\partial O$, we let $\widehat{T} \mathbf{q}_{i}:=P_{O}\left(T \mathbf{q}_{i}\right)$ in all cases. Then $\widehat{T}$ is a subdivision scheme which produces a tangential gliding motion along the obstacle boundary $\partial O$ (see Fig. 9.6). We don't go into the computational issues involved in computing $P_{O}$. In fact, "as close as possible" may well be replaced by "close enough for the purpose we have in mind". We return to $P_{O}$ when discussing smoothness in Sec. 3.

2.4.5 Kinematic chains. We may use subdivision for the modeling of motions of several rigid bodies interacting with each other. This is done by subdividing polygons in the so-called configuration space, a point of which represents the positions of the bodies in question [Latombe 2001]. Here we consider only the simple case of two bodies connected by a spherical joint: Assume that the joint is located at coordinates $\mathbf{x}$ and $\mathbf{y}$ in respective local coordinate systems attached to the two bodies. The two bodies can assume only such positions $(A, \mathbf{a})$ and $(B, \mathbf{b})$, where $A \mathbf{x}+\mathbf{a}=B \mathbf{y}+\mathbf{b}$. This is the equation of the configuration space $\mathcal{C}$ of this two-element kinematic chain:

$$
\mathcal{C}=\left\{(A, \mathbf{a}, B, \mathbf{b}) \mid A \mathbf{x}+\mathbf{a}=B \mathbf{y}+\mathbf{b},(A, \mathbf{a}) \in \mathrm{SE}_{3},(B, \mathbf{b}) \in \mathrm{SE}_{3}\right\} \subset \mathbb{R}^{d}, \quad d=3 \times 3+3+3 \times 3+3=24 .
$$

$\mathcal{C}$ is a smooth surface in $\mathbb{R}^{d}$. A way of projecting a general element $(A, \mathbf{a}, B, \mathbf{b})$ of $\mathbb{R}^{24}$ onto the configuration space is to first project both $(A, \mathbf{a})$ and $(B, \mathbf{b})$ onto elements $\left(A^{\prime}, \mathbf{a}^{\prime}\right)$ and $\left(B^{\prime}, \mathbf{b}^{\prime}\right)$ contained in the Euclidean motion group, and then to modify $\mathbf{a}^{\prime}$ and $\mathbf{b}^{\prime}$ such that the side condition is fulfilled. We may choose e.g. $\mathbf{a}^{\prime \prime}:=$ $\mathbf{a}^{\prime}+\mathbf{n}$ and $\mathbf{b}^{\prime \prime}:=\mathbf{b}^{\prime}-\mathbf{n}$ with $\mathbf{n}=\left(B^{\prime} \mathbf{y}+\mathbf{b}^{\prime}-A^{\prime} \mathbf{x}-\mathbf{a}^{\prime}\right) / 2$. Then the mapping $(A, \mathbf{a}, B, \mathbf{b}) \mapsto\left(A^{\prime}, \mathbf{a}^{\prime \prime}, B^{\prime}, \mathbf{b}^{\prime \prime}\right)$ is a smooth projection onto the configuration space, and we can do projection subdivision (see Fig. 10).

\subsection{Tensor product rules}

There is an obvious extension of the concept of curve subdivision scheme to higher dimensions - it is the tensor product rule $S \otimes S^{\prime}$ of curve subdivision rules $S, S^{\prime}$ which does not operate on polygons, but on regular grids which, combinatorially, are products of polygons. One round of subdivision by $S \otimes S^{\prime}$ means subdividing the columns of the grid with $S$, and then subdividing the rows of the resulting refined grid with 

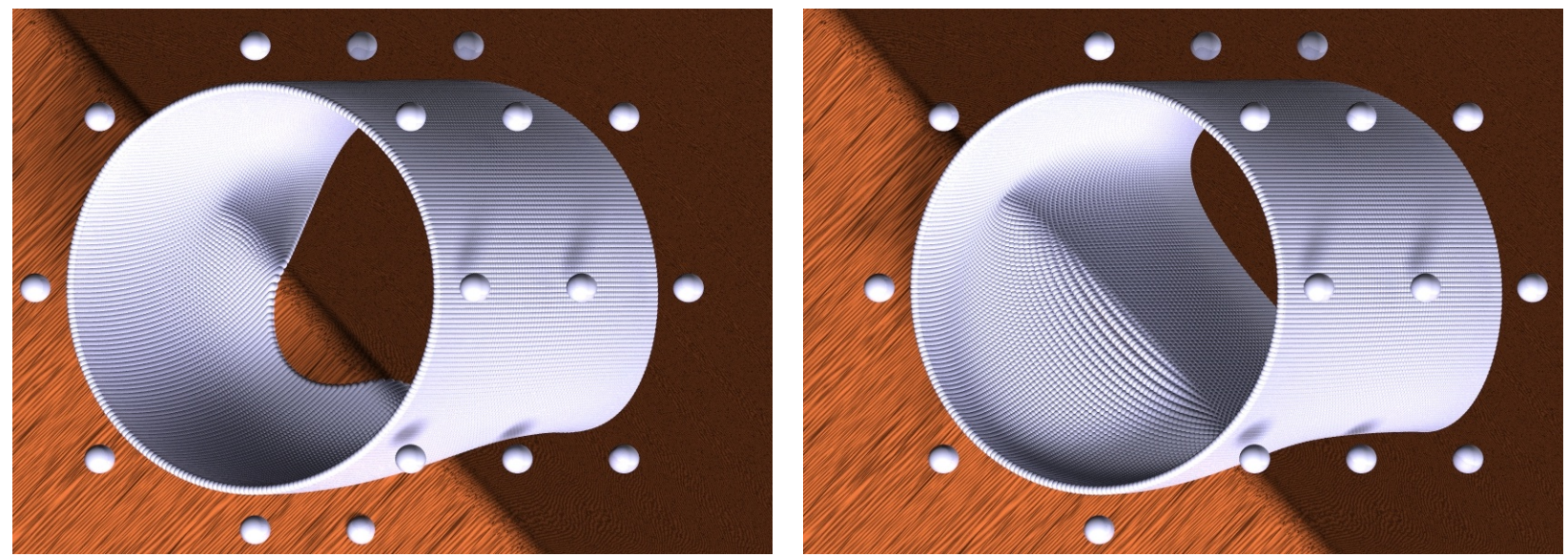

Fig. 11. Both images show a tensor product surface together with its cylindrical control grid defined by an obstacle modification of the linear scheme $B_{5} \otimes B_{5}$.

$S^{\prime}$. A picture of an obstacle modification of such a tensor product rule is shown in Fig. 11.

\subsection{A note on implementation}

Any existing implementation of a subdivision scheme based on affine averages can be "perturbed" in order to become an implementation of one of the nonlinear schemes described in the present paper. The perturbation either consists of projecting the affine average, or of replacing the affine average with a group or geodesic average. It is usually not hard to implement projections, and for group-midpoints explicit formulae are available. The computation of geodesics, however, is a topic of much previous and current research, and many solutions are available [Mémoli and Sapiro 2001; 2005; Surazhsky et al. 2005].

\section{THEORY: NONLINEAR PERTURBATIONS OF LINEAR SCHEMES}

This section first describes the concept of proximity of subdivision schemes and the smoothness results which are available from [Wallner and Dyn 2005] and [Wallner 2006a]. Afterwards we verify that the various ways of perturbing linear schemes introduced in Section 2 fulfill the requirements of the theory. This turns out to be easy for the surface and motion cases, because general results already exist.

\subsection{Notation and general results}

We use the symbol $\Delta \mathbf{p}$ for the forward difference sequence of a polygon: $\Delta \mathbf{p}_{i}=\mathbf{p}_{i+1}-\mathbf{p}_{i}$, and also the notation $\delta(\mathbf{p})=\sup _{i}\left\|\mathbf{p}_{i}-\mathbf{p}_{i+1}\right\|$. Following [Wallner and Dyn 2005; Wallner 2006a], we define that subdivision schemes $S$ and $T$ are in 0-proximity, if if there is a constant $C$ such that

$$
\sup _{i}\left\|S \mathbf{p}_{i}-T \mathbf{p}_{i}\right\|<C \delta(\mathbf{p})^{2}
$$

whenever $\delta(\mathbf{p})$ is small enough. Similarly, $S$ and $T$ are said to be in 1-proximity, if they are in 0-proximity, and in addition for small enough $\delta(\mathbf{p})$,

$$
\sup _{i}\left\|\Delta S \mathbf{p}_{i}-\Delta T \mathbf{p}_{i}\right\|<C\left(\delta(\Delta \mathbf{p}) \delta(\mathbf{p})+\delta(\mathbf{p})^{3}\right) .
$$

The following is a result of [Wallner and Dyn 2005]: Suppose that $S$ and $T$ are subdivision schemes in 0-proximity, and that $S$ is an affinely invariant linear scheme, which has certain technical properties (the ACM Transactions on Graphics, Vol. V, No. N, Month 20YY. 
B-spline schemes $B_{n}$ for $n \geq 2$ and also the interpolatory four-point scheme of Equ. (3) have them). Then $T^{\infty} \mathbf{p}$ exists for all polygons $\mathbf{p}$ with $\delta(\mathbf{p})$ small enough, and $T^{\infty} \mathbf{p}$ is a $C^{1}$ curve for all polygons $\mathbf{p}$ where subdivision converges. According to [Wallner 2006a], if $S=B_{n}$ with $n \geq 3$ and $S, T$ are in 1-proximity, then $T^{\infty} \mathbf{p}$ is a $C^{2}$ curve for all polygons $\mathbf{p}$ where subdivision converges.

\subsection{Overview of the convergence proof}

For the convenience of the reader, we repeat the proof of the convergence theorem as presented in [Wallner and Dyn 2005]. Recall that convergence of a linear subdivision scheme " $S$ " is essentially equivalent to the existence of $\mu<1$ such that

$$
\delta\left(S^{l} \mathbf{p}\right) \leq \mu^{l} \delta(\mathbf{p}) \text { for all } l, \mathbf{p} .
$$

There are linear schemes which are convergent without fulfilling (12). In that case, several rounds of $S$ have to be taken as one round of a new linear scheme, which then fulfills (12), see [Dyn 1992].

For the precise formulation of convergence, we have to assign a polyline to a sequence of vertices. For an $N$-ary subdivision scheme, this is done as follows: For points $\mathbf{p}_{i}$, the piecewise linear function " $t \mapsto F_{j}(\mathbf{p})(t)$ " assumes the value $\mathbf{p}_{i}$ exactly for $t=i / N^{j}$, and is assumed to be linear in each interval $\left[i / N^{j},(i+1) / N^{j}\right]$. It is obvious by construction that for all $j$,

$$
\sup _{i}\left\|\mathbf{p}_{i}-\mathbf{q}_{i}\right\|=\left\|\mathcal{F}_{j}(\mathbf{p})-\mathcal{F}_{j}(\mathbf{q})\right\|_{\infty}, \quad \text { where }\|f\|_{\infty}:=\sup _{t}\|f(t)\| .
$$

If subdivision is applied to $\mathbf{p}$, we get sequences $T \mathbf{p}, T^{2} \mathbf{p}, \ldots$ We consider the polylines $F_{0}(\mathbf{p}), F_{1}(T \mathbf{p})$, $F_{2}\left(T^{2} \mathbf{p}\right), \ldots$ and ask for the limit of this sequence of functions. Now the subdivision scheme $S$ is said to converge when applied to $\mathbf{p}$, if the limit of the functions $F_{j}\left(T^{j} \mathbf{p}\right)$ exists for $j \rightarrow \infty$ in the sense of the sup-norm defined by (13).

For a linear convergent scheme $S$ with finite mask the rate of convergence towards its limit is well known (cf. Equations (3.8)-(3.10) of [Dyn 1992]): There is a constant $\gamma$, depending only on $S$, such that

$$
\left\|\mathcal{F}_{l+1}(S \mathbf{q})-\mathcal{F}_{l}(\mathbf{q})\right\|_{\infty} \leq \gamma \delta(\mathbf{q}), \quad \text { for all } \mathbf{q}, l .
$$

We are now ready to formulate and prove a theorem on convergence of nonlinear subdivision schemes which are in proximity with linear ones.

TheOrem 1. Assume that $S, T$ are in 0-proximity, i.e., there is $\epsilon>0$ such that (10) holds true whenever $\delta(\mathbf{p})<\epsilon$. Further assume that the linear scheme $S$ fulfills (12). Then there is $\epsilon^{\prime}>0$ and $\bar{\mu}<1$ such than an inequality analogous to(12) is fulfilled:

$$
\delta\left(T^{l} \mathbf{p}\right) \leq \bar{\mu}^{l} \delta(\mathbf{p}) \text { for all } l, \mathbf{p} \text { with } \delta(\mathbf{p})<\epsilon^{\prime} .
$$

In that case, the sequence $T^{l} \mathbf{p}$ converges to a continuous curve.

Proof. We let $d_{l}:=\delta\left(T^{l} \mathbf{p}\right)$. By the triangle inequality, we have the implication $\sup _{i}\left\|\mathbf{p}_{i}-\mathbf{q}_{i}\right\| \leq K \Longrightarrow$ $\delta(\mathbf{p}) \leq \delta(\mathbf{q})+2 K$. This together with (10) shows that $d_{l} \leq \delta\left(S T^{l-1} \mathbf{p}\right)+2 C \delta\left(T^{l-1} \mathbf{p}\right)^{2}$. We use (12) and the definition of $d_{l}$ to get the recursion $d_{l} \leq \mu d_{l-1}+2 C d_{l-1}^{2}$. Now we choose $\epsilon^{\prime}>0$ such that $\bar{\mu}:=\mu+2 C \epsilon^{\prime}<1$. It is our aim to show that $d_{l+1} \leq \bar{\mu} d_{l}$, for all $l$. This property is referred to as " $R(l)$ ", and if it holds true for all $l$, we obviously have shown (15). " $R(0)$ " follows immediately from our choice of $\epsilon^{\prime}$ : We have $d_{1} \leq d_{0}\left(\mu+2 C d_{0}\right) \leq\left(\mu+2 C \epsilon^{\prime}\right) d_{0}=\bar{\mu}_{0} d_{0}$. In order to show " $R(l)$ " in general, we use induction. Assuming the truth of " $R(l-1)$ ", we compute $d_{l} \leq \mu d_{l-1}+2 C d_{l-1}^{2} \leq \bar{\mu}^{l-1} \delta(\mathbf{p})\left(\mu+2 C \bar{\mu}^{l-1} \delta(\mathbf{p})\right) \leq \bar{\mu}^{l-1} \delta(\mathbf{p})\left(\mu+2 C \epsilon^{\prime}\right) \leq$ $\bar{\mu}^{l} \delta(\mathbf{p})$. Thus we have " $R(l)$ " and (15).

We now use (14) and (10) together with (13) to compute $\left\|F_{j+1}\left(T^{j+1} \mathbf{p}\right)-F_{j}\left(T^{j} \mathbf{p}\right)\right\|_{\infty} \leq \| \mathcal{F}_{j+1}\left(T^{j+1} \mathbf{p}\right)-$ $\mathcal{F}_{j+1}\left(S T^{j} \mathbf{p}\right)\left\|_{\infty}+\right\| \mathcal{F}_{j+1}\left(S T^{j} \mathbf{p}\right)-\mathcal{F}_{j}\left(T^{j} \mathbf{p}\right) \|_{\infty} \leq C \delta\left(T^{j} \mathbf{p}\right)^{2}+\gamma \delta\left(T^{j} \mathbf{p}\right)$. By (15), this expression is bounded 
by a factor times $\bar{\mu}^{j}$, with $\bar{\mu}<1$. It follows that $\mathcal{F}_{j}\left(T^{j} \mathbf{p}\right)$ is a Cauchy sequence with respect to the sup-norm, i.e., the limit curve exists and is continuous.

\subsection{The proof of the smoothness results}

The proofs of the smoothness results in [Wallner and Dyn 2005] and [Wallner 2006a] are along the lines of $\S 3.2$, but considerably more complicated. For an $N$-ary subdivision scheme $T$, they aim at inequalities of the following form, which for $k=0$ is similar to (12):

$$
\delta\left(N^{j l} \Delta^{j} S^{l} \mathbf{p}\right) \leq \mu_{j}^{l} P_{j}(l) \delta(\mathbf{p}), \quad j=1, \ldots, k,
$$

for some $k$, where $P_{j}$ is a nonnegative polynomial. This inequality is the key ingredient in showing $C^{k}$ smoothness of the limit curves $\lim _{j \rightarrow \infty} \mathcal{F}_{j}\left(T^{j} \mathbf{p}\right)$. For details, the interested reader is referred to the papers mentioned above.

\subsection{Verifying proximity}

We apply the general results of the previous paragraphs to the nonlinear schemes encountered in Section 2. In each case we have to show that a nonlinear scheme $T$ and a linear scheme $S$ fulfill the proximity inequality (10) for $C^{1}$ smoothness and in addition the inequality (11) for $C^{2}$ smoothness.

While it is usually not difficult to show (10) in the various cases where it holds true, the currently available proofs of (11) for both geodesic and projection subdivision occupy 13 densely written pages in [Wallner 2006a].

Geodesic subdivision. It is shown in [Wallner and Dyn 2005] that a geodesic subdivision scheme $T$ is always in 0-proximity with the linear scheme $S$ it is derived from; provided the surfaces and manifolds involved are $C^{2}$. Together with the results of Sec. 3.1, this means that the geodesic subdivision rules in surfaces and Riemannian manifolds produce $C^{1}$ limits. Wallner and Dyn [2006a] show that for the B-spline schemes $B_{n}$ with $n \geq 3$ we have even 1-proximity (provided the surfaces and manifolds involved are $C^{3}$ ), and therefore geodesic B-spline subdivision has $C^{2}$ limit curves.

Helical subdivision. According to [Wallner and Dyn 2005; Wallner 2006a] the results concerning $C^{1}$ and $C^{2}$ smoothness of geodesic subdivision extend to helical subdivision in $\mathrm{SE}_{3}$.

Projection subdivision. Again according to [Wallner and Dyn 2005; Wallner 2006a] the results concerning $C^{1}$ and $C^{2}$ smoothness of geodesic subdivision extend to nonlinear schemes defined in terms of a projection onto a surface; provided after some rounds of subdivision all edges of polygons do not leave a neighbourhood of the surface where the projection is smooth and has bounded derivatives up to second order (for $C^{1}$ smoothness) or third order (for $C^{2}$ smoothness). These conditions are fulfilled for the projection onto $\mathrm{SE}_{3}$ employed in Section 2.4.2.

Obstacle rules for curves. An obstacle rule is a kind of one-sided projection rule. Consider an obstacle $O$, its boundary surface $\partial O$, and the projection $P$ onto $\partial O$. Suppose that $S$ is a linear rule, $T$ is the projection rule, and $\widetilde{T}$ is the obstacle rule corresponding to $S$. By the previous discussion, $S$ and $T$ are in 0-proximity. As $T \mathbf{p}_{i}$ either equals $T \mathbf{p}_{i}$ or $S \mathbf{p}_{i}$, it is trivial that also $S$ and $\widetilde{T}$ are in 0 -proximity. It follows that the results concerning $C^{1}$ smoothness of projection rules extend to obstacle rules.

"Arbitrary" perturbations. This title refers to perturbations like the one described by (5) in Sec. 2.1, which are not entailed by the underlying geometry.

0-proximity is obviously transitive in the following sense: If $S$ and $T$ are in 0-proximity, and so are $T$ and $U$, then also the schemes $S$ and $U$ are in 0-proximity. This means that further perturbing a nonlinear scheme such that the magnitude of perturbation is bounded by a constant times $\left\|\mathbf{p}_{i+1}-\mathbf{p}_{i}\right\|^{2}$ does not 
destroy 0-proximity, and therefore does not influence $C^{1}$ smoothness. The gravitational effect introduced by (4) is an example of this.

Subdivision rules which generate motions in the presence of obstacles. Using the concept of configuration space, we are able to reduce these cases to the previous ones. The set of positions of a rigid body such that it intersects a given obstacle $O$, is a subset of $\mathrm{SE}_{3}$ denoted by $\widetilde{O}\left(\mathrm{SE}_{3}\right.$ minus $\widetilde{O}$ then is the configuration space of moving outside the obstacle). Its boundary $\partial \widetilde{O}$ consists of the set of positions such that the given rigid body touches $\partial O$ without intersecting the interior of $O(\partial \widetilde{O}$ is the configuration space of gliding on $O)$. Computing $P_{O}(A, \mathbf{a})$ means projecting $(A, \mathbf{a})$ onto $\partial \widetilde{O}$. Thus the subdivision process under consideration is nothing but an obstacle scheme (if we don't have a gliding constraint) or a projection scheme (if we consider gliding on $O$ ). The smoothness results apply, if $P_{O}$ is smooth enough. The smoothness of $P_{O}$ depends on the surfaces involved and on the exact definition of $P_{O}$. We do not deal with this topic here, as it would lead too far. The obstacle in Fig. 9.3-5 is only piecewise smooth - and so are both $P_{O}$ and the limit motion.

Tensor product rules. The smoothness of nonlinear tensor product rules does not follow in a straightforward way from the results of on curve subdivision and is the topic of [Wallner 2006b]. There it is shown that the tensor product rules, if some technical conditions are fulfilled, have essentially the same smoothness properties as curve subdivision rules. The surfaces shown in Fig. 11 are $C^{1}$.

\section{PERTURBATION BY COMPUTATION}

The very aim of Computer Graphics - visualization - often makes speed of computations more important than their accuracy. It is therefore important to know that smoothness of a limit is stable with respect to sloppiness in computations.

Suppose that a linear subdivision scheme $S$ and a nonlinear scheme $T$ satisfy the requirements cited above which ensure that $T$ 's limit curves are $C^{1}$, but $T$ is cumbersome to compute and therefore is replaced by an approximation $\bar{T}$. The limit curves of $\bar{T}$ are $C^{1}$ also, if $T$ and $\bar{T}$ are in 0-proximity, because then so are $S$ and $\bar{T}$. This section shows that this principle applies to the discretization of the concept of geodesic line and the incomplete computation of such discrete geodesics. It can be seen as a model for the treatment of other differential equations.

\subsection{Computation of discrete geodesics}

Section 2.2.4 discusses how to compute a discrete geodesic $\mathbf{p}_{0}, \ldots, \mathbf{p}_{k}$ with given endpoints $\mathbf{a}=\mathbf{p}_{0}$ and $\mathbf{b}=\mathbf{p}_{k}$. For the use in subdivision, we suggest that $k$ is even (e.g. $k=2$ or $k=4$ ), because then $\mathbf{p}_{k / 2}$ serves as discrete geodesic midpoint. For evaluating the Chaikin rule, we have to discretize both $g-a_{1 / 4}$ and $\mathrm{g}-\mathrm{av}_{3 / 4}$ : We choose $k$ as a multiple of four, and use the points $\mathbf{p}_{k / 4}$ and $\mathbf{p}_{3 k / 4}$, respectively.

Minimizing the discrete energy mentioned in Sec. 2.2.4 can be done by the numerical optimization algorithm of your choice. Usually such an algorithm works by first finding an initial guess, which is improved iteratively. We are interested in the order of accuracy of such a method. For simplicity, we consider only the case that $k$ is even, and we assume that the subdivision algorithm is defined solely using geodesic midpoints (because of Equ. (7), this includes all B-spline rules.). We assume that $\mathbf{p}_{k / 2}$ is the exact discrete geodesic midpoint, and that $\mathbf{q}_{k / 2}$ is the point computed by some algorithm which we don't want to specify, but which is required to meet two conditions. The first one is as follows: Assume that the given surface is parametrized by some function $\mathbf{f}(\mathbf{u})$, where $\mathbf{u}$ is a list of surface parameters (e.g. $\mathbf{u}=(u, v)$ ), and that $\mathbf{a}=\mathbf{f}\left(\mathbf{u}_{0}\right), \mathbf{b}=\mathbf{f}\left(\mathbf{u}_{1}\right)$. With $\mathbf{r}_{i}:=\mathbf{f}\left(\mathbf{u}_{0}+i\left(\mathbf{u}_{1}-\mathbf{u}_{0}\right) / k\right)$, we use $\mathbf{r}_{0}, \ldots, \mathbf{r}_{k}$ as initial polygon of our iteration.

The second condition which our iterative algorithm has to satisfy is that its result $\mathbf{q}_{k / 2}$ of computation must not be far from both the initial value $\mathbf{r}_{k / 2}$ and the exact result $\mathbf{p}_{k / 2}$. So we assume that there is a constant $D$ with $\left\|\mathbf{r}_{k / 2}-\mathbf{q}_{k / 2}\right\| \leq D\left\|\mathbf{r}_{k / 2}-\mathbf{p}_{k / 2}\right\|$. This assumption is reasonable and rather weak. It will 
certainly be fulfilled for iterative procedures where $\mathbf{q}_{k / 2}$ actually converges to $\mathbf{p}_{k / 2}$.

\subsection{General remarks on the discretization of ordinary differential equations}

The numerical theory of ODEs has been a standard topic of numerical analysis for a long time. As this paper needs only the differential equation of geodesics, we here consider second order ODEs which have the implicit form $F(t, y(t), \ddot{y}(t))=0$ with $y$ and $F$ as smooth vector-valued functions. The function $F$ is given, and $y(t)$ is unknown except for $y\left(t_{0}\right)$ and $\dot{y}\left(t_{0}\right)$. We choose a time step $\Delta t$, so that $t_{j}=t_{0}+j \Delta t$ $(j=0,1,2, \ldots)$ and discretize this ODE by the definitions $y_{0}=y\left(t_{0}\right), y_{1}=y_{0}+\Delta t \dot{y}\left(t_{0}\right)$, and letting $F\left(t_{j}, y_{j},\left(y_{j+1}-2 y_{j}+y_{j-1}\right) / \Delta t^{2}\right)=0$ for $j=2,3, \ldots$ Thus solving the ODE is reduced to the (possibly numerically hard) solution of a finite system of equations. This is a so-called implicit Euler method. The error we make in a fixed number " $k$ " of steps is bounded as follows:

$$
\left\|y\left(t_{0}+j \Delta t\right)-y_{j}\right\| \leq \widehat{C} \cdot \Delta t^{2}, j=0, \ldots, k \text {, where } \widehat{C} \text { depends on } F, y\left(t_{0}\right), \dot{y}\left(t_{0}\right) \text {, and } k \text {. }
$$

The constant $\widehat{C}$ depends on $y\left(t_{0}\right), \dot{y}\left(t_{0}\right)$ in a continuous way. Thus for any compact set where those values are picked from, there is a global constant $\widehat{C}$. When discretizing the differential equation of geodesics we make use of this fact: We require that geodesic lines are traversed with unit speed, i.e., the initial conditions "cc $(0)=\mathbf{p}, \dot{\mathbf{c}}(0)=\mathbf{v}$ " are always such that $\|\mathbf{v}\|=1$. As long as we pick $\mathbf{p}$ from a compact set, the total set of pairs $(\mathbf{p}, \mathbf{v})$ is compact, and there is a global upper bound $\widehat{C}$ for the discretization error in (17).

\subsection{Error estimates}

We consider a linear subdivision rule expressible in terms of midpoints. Its geodesic analogue employs geodesic midpoints. As this rule is discretized, we use a discrete geodesic midpoint instead (the point $\mathbf{p}_{k / 2}$ from above). As we don't want to spend too much time computing that point, we use $\mathbf{q}_{k / 2}$ instead of $\mathbf{p}_{k / 2}$. Our aim is to establish that the resulting subdivision scheme is in 0 -proximity with the original one, so that we still have $C^{1}$ smoothness.

TheOREM 2. A linear subdivision rule expressible via midpoints is in 0-proximity with its geodesic/discrete/numerical analogue as described above.

Proof. Part 1: We have to show that for any $\mathbf{a}$ and $\mathbf{b}$, the distance $\left\|\mathbf{q}_{k / 2}-\operatorname{av}_{1 / 2}(\mathbf{a}, \mathbf{b})\right\|$ is bounded by a constant times $\|\mathbf{a}-\mathbf{b}\|^{2}=: \delta^{2}$, provided $\delta$ is small enough. According to [Wallner and Dyn 2005], such a constant $C$ exists for the distance of the actual geodesic midpoint $g$-av $\operatorname{av}_{1 / 2}(\mathbf{a}, \mathbf{b})$ from $\operatorname{av}_{1 / 2}(\mathbf{a}, \mathbf{b})$ - this is the fact that a linear subdivision scheme is in 0-proximity with its geodesic analogue. We are going to establish that such a constant $C^{\prime}$ exists also for the distance of $\operatorname{g}^{-a_{1 / 2}}(\mathbf{a}, \mathbf{b})$ from $\mathbf{p}_{k / 2}$ - this is the discretization error (Part 2 of the proof). Part 3 below shows that there is an analogous constant $C^{\prime \prime}$ for the distance $\left\|\operatorname{av}_{1 / 2}(\mathbf{a}, \mathbf{b})-\mathbf{r}_{k / 2}\right\|$. We now assume that the constants $C, C^{\prime}, C^{\prime \prime}$ at our disposal. Then each of the distances

$$
\mathbf{r}_{k / 2}-\operatorname{av}_{1 / 2}(\mathbf{a}, \mathbf{b})-\mathrm{g}_{-\mathrm{av}_{1 / 2}}(\mathbf{a}, \mathbf{b})-\mathbf{p}_{k / 2}
$$

has a known bound, implying that $\left\|\mathbf{r}_{k / 2}-\mathbf{p}_{k / 2}\right\| \leq \delta^{2} \cdot\left(C+C^{\prime}+C^{\prime \prime}\right)$. It follows that $\left\|\mathbf{r}_{k / 2}-\mathbf{q}_{k / 2}\right\| \leq$ $\delta^{2} D\left(C+C^{\prime}+C^{\prime \prime}\right)$. The known bounds for the distances

$$
\mathbf{q}_{k / 2}-\mathbf{r}_{k / 2}-\operatorname{av}_{1 / 2}(\mathbf{a}, \mathbf{b})
$$

show that $\left\|\mathbf{q}_{k / 2}-\operatorname{av}_{1 / 2}(\mathbf{a}, \mathbf{b})\right\| \leq \delta^{2} \cdot\left(D\left(C+C^{\prime}+C^{\prime \prime}\right)+C^{\prime \prime}\right)$, which concludes the proof of 0-proximity.

Part 2: Section 2.2.4, which describes discrete geodesic lines, is actually the application of the general concept of Section 4.2 to the differential equation of geodesics. We therefore have the error estimate of (17). Unfortunately (17) is formulated in terms of the discretization time step $\Delta t$ rather than in terms of $\|\mathbf{a}-\mathbf{b}\|$ as required in our proof. This problem is resolved as follows: The curvature radius $\rho(s)$ of 
a geodesic line $\mathbf{c}(s)$ is at least $\rho_{\min }=1 / \kappa$, where $\kappa$ is an upper bound for the normal curvatures of the surface in question [do Carmo 1992]. It is an elementary fact that for any such geodesic arc of length $L$, we have the inequality $L \geq\|\mathbf{c}(0)-\mathbf{c}(L)\| \geq L / \pi$, provided $L \leq \pi \rho_{\min }$ (for a semicircle of radius $\rho_{\min }$, the second inequality is sharp). As geodesics are traversed by unit velocity, the length $L$ of the geodesic equals $k \Delta t$, and we have $\Delta t \leq \pi\|\mathbf{a}-\mathbf{b}\| / k$. This allows to re-formulate the error term of (17) in the form $\left\|\mathrm{g}-\mathrm{av}_{1 / 2}(\mathbf{a}, \mathbf{b})-\mathbf{p}_{k / 2}\right\| \leq C^{\prime}\|\mathbf{a}-\mathbf{b}\|^{2}$, as required.

Part 3: As to the existence of $C^{\prime \prime}$, we consider the curve $\mathbf{d}(t)=\mathbf{f}\left(\mathbf{u}_{0}+t\left(\mathbf{u}_{1}-\mathbf{u}_{0}\right)\right)$. By construction, $\mathbf{a}=\mathbf{d}(0), \mathbf{r}_{k / 2}=\mathbf{d}(1 / 2), \mathbf{b}=\mathbf{d}(1), \operatorname{av}_{1 / 2}(\mathbf{a}, \mathbf{b})=(\mathbf{d}(0)+\mathbf{d}(1)) / 2$. By Taylor's theorem, $\mathbf{d}(t)=\mathbf{d}(0)+$ $t \mathbf{d}^{\prime}(0)+t^{2} \mathbf{d}^{\prime \prime}\left(\theta_{t}\right) / 2$, where $0 \leq \theta_{t} \leq t$. We plug Taylor's formula into the previous expressions and find that

$$
\mathbf{r}_{k / 2}-\operatorname{av}_{1 / 2}(\mathbf{a}, \mathbf{b})=\mathbf{d}^{\prime \prime}\left(\theta_{1 / 2}\right) / 8-\mathbf{d}^{\prime \prime}\left(\theta_{1}\right) / 4 \text {. }
$$

We easily verify that the $i$-th component of $\mathbf{d}^{\prime \prime}(t)$ is computed by $\mathbf{d}_{i}^{\prime \prime}(t)=\left(\mathbf{u}_{1}-\mathbf{u}_{0}\right)^{T} \cdot H_{i}(t) \cdot\left(\mathbf{u}_{1}-\mathbf{u}_{0}\right)$, where $H_{i}(t)$ is the matrix of second derivatives of the $i$-th component $\mathbf{f}_{i}$ of the given surface parametrization in the point $\mathbf{d}(t)$. Those are bounded for a compact piece of surface, so it follows that $\mathbf{d}^{\prime \prime}(t)$, and therefore also $\left\|\mathbf{r}_{k / 2}-\operatorname{av}_{1 / 2}(\mathbf{a}, \mathbf{b})\right\|$ is bounded by a constant times $\left\|\mathbf{u}_{1}-\mathbf{u}_{0}\right\|^{2}$. This is almost what we wanted to show. Actually we need a constant times $\|\mathbf{a}-\mathbf{b}\|^{2}$. So it remains to find $D^{\prime}$ such that $\left\|\mathbf{u}_{1}-\mathbf{u}_{0}\right\| \leq D^{\prime}\|\mathbf{a}-\mathbf{b}\|$. The meaning of this inequality is that if points of a surface are close together, so are their respective parameter values. We rephrase the last condition to $\left\|\mathbf{u}_{1}-\mathbf{u}_{0}\right\| /\left\|\mathbf{f}\left(\mathbf{u}_{1}\right)-\mathbf{f}\left(\mathbf{u}_{0}\right)\right\| \leq D^{\prime}$ and show that the left hand expression has a maximum, then equal to $D^{\prime}$. This follows (by compactness of the surface) from continuity of that expression: Continuity is trivial for $\mathbf{u}_{1} \neq \mathbf{u}_{2}$ and follows from smoothness of $\mathbf{f}$ if $\mathbf{u}_{1}$ approaches $\mathbf{u}_{2}$. The proof is complete.

\section{CONCLUSION}

We have shown several natural analogues of curve subdivision rules in nonlinear geometries. All of them are perturbations of linear rules, but still close enough so that smoothness can be shown. The types of perturbation range from geodesic instead of affine subdivision, moving points out of obstacles, projecting points onto fixed surfaces, and perturbation by sloppy computation. The available theory shows that many ways of intuitively placing control handles and subdividing actually leads to smooth limits.

\section{ACKNOWLEDGMENTS}

The authors would like to express their thanks to Nira Dyn for many fruitful discussions.

\section{REFERENCES}

Alekseevskij, D. V., Vinberg, E. B., And Solodovnikov, A. S. 1993. Geometry of spaces of constant curvature. In Geometry, II. Encyclopaedia Math. Sci., vol. 29. Springer, Berlin, 1-138.

Belta, C. And Kumar, V. 2002. An SVD-based projection method for interpolation on SE(3). IEEE Trans. Robotics Automation 18, 334-345.

Cohen, A., Dyn, N., And Matei, B. 2003. Quasilinear subdivision schemes with applications to ENO interpolation. Appl. Comput. Harmon. Anal. 15, 89-116.

do Carmo, M. P. 1992. Riemannian Geometry. Birkhäuser Verlag, Boston Basel Berlin.

Donoho, D. L. 2001. Wavelet-type representation of Lie-valued data. Talk at the IMI "Approximation and Computation" meeting, May 12-17, 2001, Charleston, South Carolina.

Dyn, N. 1992. Subdivision schemes in CAGD. In Advances in Numerical Analysis Vol. II, W. A. Light, Ed. Oxford University Press, Oxford, 36-104.

Dyn, N., Gregory, J., And Levin, D. 1987. A four-point interpolatory subdivision scheme for curve design. Computer Aided Geometric Design 4, 257-268.

Higham, N. J. 1989. Matrix nearness problems and applications. In Applications of matrix theory (Bradford, 1988). Inst. Math. Appl. Conf. Ser. New Ser., vol. 22. Oxford Univ. Press, New York, 1-27. 
Jüttler, B. And Wagner, M. 2002. Kinematics and animation. In Handbook of Computer Aided Geometric Design, G. Farin, J. Hoschek, and M.-S. Kim, Eds. North Holland, Amsterdam, 723-748.

Kim, M.-J., Kim, M.-S., And Shin, S. 1995. A general construction scheme for unit quaternion curves with simple high order derivatives. In Proceedings of SIGGRAPH 95. Computer Graphics Proceedings, Annual Conference Series. ACM, ACM Press/ACM SIGGRAPH, New York, 369-376.

Kobbelt, L. 2002. Multiresolution techniques. In Handbook of Computer Aided Geometric Design, G. Farin, J. Hoschek, and M.-S. Kim, Eds. North-Holland, Amsterdam, 343-361.

Latombe, J. C. 2001. Robot Motion Planning. Kluwer, Boston.

Marinov, M., Dyn, N., And Levin, D. 2004. Geometrically controlled 4-point interpolatory schemes. In Advances in Multiresolution for Geometric Modelling, N. Dodgson, M. S. Floater, and M. Sabin, Eds. Springer, Berlin, 301-317.

MÉmoli, F. And Sapiro, G. 2001. Fast computation of weighted distance functions and geodesics on implicit hyper-surfaces. J. Comput. Phys. 173, 2, 730-764.

MÉmoli, F. And Sapiro, G. 2005. Distance functions and geodesics on submanifolds of $\mathbb{R}^{d}$ and point clouds. SIAM J. Appl. Math. 65, 4, 1227-1260.

NoAKes, L. 1997. Riemannian quadratics. In Curves and Surfaces with Applications in CAGD. Vol. 1. Vanderbilt University Press, Nashville and London, 319-328.

NoAkes, L. 1998. Non-linear corner cutting. Advances Comp. Math. 8, 165-177.

NoAkes, L. 1999. Accelerations of Riemannian quadratics. Proc. American Math. Soc. 127, 1827-1836.

Oswald, P. 2004. Smoothness of nonlinear median-interpolation subdivision. Advances Comput. Math. 20, 401-423.

Ramamoorthi, R. AND BARR, A. 1997. Fast construction of accurate quaternion splines. In Proceedings of ACM SIGGRAPH 97. Computer Graphics Proceedings, Annual Conference Series. ACM, New York, 287-292.

Sabin, M. A. 2002. Interrogation of subdivision surfaces. In Handbook of Computer Aided Geometric Design, G. Farin, J. Hoschek, and M.-S. Kim, Eds. North-Holland, Amsterdam, 327-341.

Sprott, K. And Ravani, B. 1997. Ruled surfaces, Lie groups and mesh generation. In Proc. ASME Design Eng. Techn. Conf. 1997. ASME, New York, No. DETC97/DAC-3966.

Surazhsky, V., Surazhsky, T., Kirsanov, D., Gortler, S. J., And Hoppe, H. 2005. Fast exact and approximate geodesics on meshes. ACM Trans. Graphics 24, 3, 553-560. (Proceedings of ACM SIGGRAPH 2005).

Ur Rahman, I., Drori, I., Stodden, V. C., Donoho, D. L., And Schröder, P. 2005. Multiscale representations for manifoldvalued data. Multiscale Modeling and Simulation 4, 4, 1201-1232.

Wallner, J. 2006a. Smoothness analysis of subdivision schemes by proximity. Constr. Approx.. to appear.

WALlner, J. 2006b. Smoothness of nonlinear tensor product subdivision schemes. forthcoming paper.

Wallner, J. And Dyn, N. 2005. Convergence and $C^{1}$ analysis of subdivision schemes on manifolds by proximity. Comput. Aided Geom. Design 22, 7, 593-622.

Warren, J. And Weimer, H. 2001. Subdivision Methods for Geometric Design: A Constructive Approach. Morgan Kaufmann Series in Computer Graphics. Morgan Kaufmann, San Francisco.

XIE, G. ANd YU, T. P.-Y. 2005. Smoothness analysis of nonlinear subdivision schemes of homogeneous and affine invariant type. Constr. Approx. 22, 2, 219-254. 